\title{
Almost Periodic Jacobi Matrices Associated with Julia Sets for Polynomials
}

\author{
M. F. Barnsley ${ }^{1}$, J. S. Geronimo ${ }^{2}$, and A. N. Harrington ${ }^{2,3}$ \\ School of Mathematics, Georgia Institute of Technology, Atlanta, Georgia 30332, USA
}

\begin{abstract}
Let $\mathscr{J}$ be the Jacobi matrix associated with polynomial $T(z)$ of degree $N \geqq 2$. The spectrum of $\mathscr{J}$ is the Julia set associated with $T(z)$ which in many cases is a Cantor set. Let $\mathscr{J}^{(1)}$ denote the result of omitting the first row and column of $J$. Then it is shown that the spectrum of $\mathscr{J}^{(1)}$ may be purely discrete.

It is also shown that for $T(z)=\alpha^{N} C_{N}(z / \alpha)$ for $\alpha>\sqrt{3 / 2}$, where $C_{N}$ is a Chebychev polynomial the coefficients of $\mathscr{J}$ and $\mathscr{J}^{(1)}$ are limit periodic extending the work of Bellissard, Bessis, and Moussa (Phys. Rev. Lett. 49, 701-704 (1982)).
\end{abstract}

\section{Introduction}

Let $\mathbb{C}$ be the complex plane and let $T: \mathbb{C} \rightarrow \mathbb{C}$ be a polynomial; $T(z)=z^{N}+$ $k_{1} z^{N-1}+\cdots+k_{N}$, where $N \geqq 2$ and $k_{l} \in \mathbb{C}$. Define the iterates of $T$ by $T^{0}(z)=z$ and $T^{n}(z)=T \circ T^{n-1}(z)$ for $n \in \mathbb{N}=\{1,2,3, \ldots\}$. Let $J$ be the Julia set for $T$, $[1,2,3]: J$ can be defined as the closure of the set of repulsive cycles of $T$. When $J$ is a subset of the real line $\mathbb{R}$, which is the case in this paper, then $J$ is either a generalized Cantor set with Lebesgue measure zero or it is an interval. Let $\mu$ be the balanced $T$-invariant probability measure [4] on $J$. (If $\left\{T_{i}^{-1}(z)\right\}_{i=1}^{N}$ is a complete assignment of branches of the inverse of $T$, then $\mu\left(T_{i}^{-1}(E)\right)=\mu(E) / N$ whenever $E$ is a Borel subset of $\mathbb{C}$. Equivalently, for all $f \in L_{1}(\mu, \mathbb{C})$, we have

$$
\left.\int f(z) d \mu(z)=\frac{1}{N} \int \sum_{i=1}^{N} f\left(T_{i}^{-1}(z)\right) d \mu(z) .\right)
$$

Let $\left\{P_{n}(z)\right\}_{n=0}^{\infty}$ be the monic polynomials orthogonal with respect to $\mu$; that is $P_{n}(z)$ is of degree $n$, with unit leading coefficient, and

$$
\int_{J} P_{l}(z) \overline{P_{m}(z)} d \mu(z)=0
$$

when $l \neq m$. The bar means the complex conjugate. The relationship between the

1 Supported in part by N.S.F. grant DMS-8401609

2 Supported in part by N.S.F. grant MCS- 8203325

3 Present address: Dept. of Mathematical Sciences, Loyola University, Chicago, IL 60627, USA 
orthogonal polynomials and the iterates of $T$ is now well-understood $[4,5,6]$ and can be summarized as follows:

$$
\begin{gathered}
P_{1}(z)=z+k_{1} / N, \\
P_{l N}(z)=P_{l}(T(z)), \quad l \in \mathbb{N}_{0}=\{0,1,2, \ldots,\} .
\end{gathered}
$$

Formally one can associate with $\mu$ a semi-infinite tridiagonal matrix operator $\mathscr{J}$

$$
\mathscr{J}=\left[\begin{array}{ccc}
b(0) & c(1) & 0 \\
c(1) & b(1) & c(2) \\
0 & c(2) & b(2) \cdot
\end{array}\right]
$$

where we define, when $J \subset \mathbb{R}$,

$$
b(l)=\frac{\left\langle x P_{l}^{2}\right\rangle}{\left\langle P_{l}^{2}\right\rangle}, \quad\left(l \in \mathbb{N}_{0}\right) ; \quad a(m)=c(m)^{2}=\frac{\left\langle P_{m}^{2}\right\rangle}{\left\langle P_{m-1}^{2}\right\rangle}, \quad(m \in \mathbb{N}) ;
$$

with

$$
\langle f\rangle=\int_{J} f(x) d \mu(x) .
$$

We define $a(n)=0$ and $b(n-1)=0$ whenever $n \leqq 0$. When $J \not \subset \mathbb{R}$, the coefficients of $\mathscr{J}$ are defined by analytic continuation in parameter space, starting from a polynomial transformation $T$ whose Julia set is real.

When $J \subset \mathbb{R}$ it is known from general principles that $\mathscr{J} \in \mathscr{B}\left(l_{2}^{+}\right)$(bounded linear operators on $l_{2}^{+}$, where

$$
\psi \in l_{2}^{+} \Leftrightarrow \sum_{n=0}^{\infty}|\psi(n)|^{2}<(\infty),
$$

and that $\mathscr{J}$ is self-adjoint with spectrum $J$ and spectral density $\mu$ [7].

One would like to know how the spectrum changes if $\mathscr{J}$ changes. To this end we consider $\mathscr{J}^{(1)}$ which is derived from $\mathscr{J}$ by omitting the first row and column. We find that the spectrum changes dramatically from a singular continuous spectrum to a spectrum which may be purely discrete.

Another thing one would like to answer is how the properties of $J$ are reflected in the properties of $\mathscr{J}$. It is known $[5,8]$ that there are special recurrence relations among the coefficients in $\mathscr{J}$; in an interesting recent paper, Bellissard et al. [9] have demonstrated that when $T$ is a quadratic polynomial these relations imply that the coefficients in $\mathscr{J}$ form a limit periodic sequence, under appropriate conditions. We believe that this almost periodic behavior is generic, and in support of this we report almost periodicity results in connection with the family $T(z)=\alpha^{N} C_{N}(z / \alpha)$, where $\alpha \geqq 1$ and $C_{N}$ is the monic Chebychev polynomial of degree $N \geqq 2$, orthogonal on $[-2,2]$. We comment briefly on the families $T(z)=$ $\alpha^{4} C_{4}(z / \alpha)+\beta$ and $T(z)=\alpha^{3} C_{3}(z / \alpha)+\beta, \alpha, \beta \in \mathbb{R}$.

Recently, the general problem of determining the spectrum of doubly-infinite Jacobi matrices has received a great deal of attention [10-16] because of its connection with certain quantum mechanical problems. In these problems one begins with the coefficients in the matrix and tries to deduce the nature of the spectrum. Here we are in fact considering the inverse problem: given the spectrum 
(the Julia set) deduce the properties of the coefficients. The examples considered in this paper are of special interest because much is known about the spectral measure $\mu$ and the asymptotics of the wave function [17-19].

We proceed as follows: in Sect. II we demonstrate the fact that under certain conditions the spectrum of $\mathscr{J}^{(1)}$ is purely discrete. Then in Sect. III we derive results concerning the almost periodic nature of the coefficients in the Jacobi matrix associated with the polynomial transformations discussed above. Finally (Sect. IV) we discuss some physical consequences of these results.

\section{The Spectrum of $\mathscr{J}^{(1)}$}

Let $\mathbb{C}$ be the complex plane and $\hat{C}=\mathbb{C} \cup\{\infty\}$. Let $T(z)=z^{N}+k_{1} z^{N-1}+\cdots$, $N \geqq 2$, and $\mu$ be the balanced measure associated with $T$, then the Stieltjes transform, $G(z)$, of $\mu$,

$$
G(z)=\int \frac{d u}{z-x}
$$

is an analytic function of $z$ for $z \notin J$. Furthermore from (I.1) it follows that $G(z)$ obeys the following functional relation,

$$
G(z)=\frac{T^{\prime}(z)}{N} G(T z)
$$

We now assume that $J \subset I=[b, a]$, where $I$ is the smallest real interval containing $J$. Then $\mathscr{J}$ as given in (I.5) is self-adjoint with $\mu$ as its spectral measure. Let $\mathscr{J}^{(1)}$ be derived from $J$ by omitting the first row and column and let $\mu^{(1)}$ be the spectral measure associated with $\mathscr{J}^{(1)}$.

Theorem 1. Let $G^{(1)}(z)$ be the Stieltjes transform associated with the spectral measure $\mu^{(1)}$, then $G^{(1)}(z)$ is a meromorphic function in $\hat{C} / J$ with the representation

$$
G^{(1)}(z)=\sum_{i=0}^{\infty} R\left(T^{i}(z)\right) \prod_{j=0}^{i} 1 / Q\left(T^{j}(z)\right) .
$$

Here

$$
Q(z)=\frac{T^{\prime}(z)}{N} \quad \text { and } \quad R(z)=\frac{(z-b(0)) Q(z)-(T z-b(0))}{a(1)} .
$$

Proof. Since the Julia set $J$ is bounded, $\mathscr{J}$ and a fortiori $\mathscr{J}^{(1)}$ are bounded operators. Consequently, $G^{(1)}(z)$ is analytic outside some interval $\hat{I}$ containing $\mathscr{J}$ and $z G^{(1)}(z)=O(1)$. Comparing the continued fraction expansion of $G^{(1)}(z)$ with that of $G(z)$ and using (II.2) it follows that

$$
G^{(1)}(z)=\frac{1}{Q(z)} G^{(1)}(T z)+\frac{R(z)}{Q(z)} .
$$

Iterating the above equation one finds that for $z$ large enough

$$
\left.G^{(1)}(z)=\sum_{i=0}^{n} R\left(T^{i} z\right) \prod_{j=0}^{i} 1 / Q\left(T^{j}(z)\right)+\prod_{j=0}^{n} 1 / Q\left(T^{j}(z)\right)\right) G\left(T^{n+1}(z)\right) .
$$


Since $T(z), Q(z)$, and $R(z)$ are monic polynomials of degree $N, N-1$, and $N-2$ respectively, there exists a $k>4$ such that for $|z|>k, \frac{1}{2}|z|^{N}<|T z|<2|z|^{N}$, $\frac{1}{2}|z|^{N-1}<|Q(z)|<2|z|^{N-1}, \quad$ and $\quad \frac{1}{2}|z|^{N-2}<|R(z)|<2|z|^{N-2}$. Consequently

$$
\begin{aligned}
& 2^{-\left(N^{i}-1 / N-1\right)}|z|^{N^{i}}<\left|T^{i}(z)\right|<2^{N^{i}-1 / N-1}|z|^{N^{i}}, \\
& \left|R\left(T^{i}(z)\right)\right|<2^{\left(N^{i}-1 / N-1\right)(N-2)+1}|z|^{N^{l+1}-2 N^{i}},
\end{aligned}
$$

and

$$
\prod_{j=0}^{i}\left|Q\left(T^{j}(z)\right)\right|>2^{-\left(N^{i+1}-1 / N-1\right)}|z|^{N^{+1}-1}>2^{2 N^{i+2}-3 N^{i+1}-2 N+3 / N-1},
$$

from which it follows that

$$
\left|\frac{R\left(T^{i}(z)\right)}{\prod_{j=0}^{i} Q\left(T^{2}(z)\right)}\right|<2^{2 N^{i}}|z|^{-2 N^{i}+1}
$$

Thus for $|z|>k$ one can pass to the limit $n \rightarrow \infty$ in (II.6) giving (II.3). Now using (II.3) as an analytic representation of $G^{(1)}(z)$, one finds, since $\left|T^{m}(z)\right|>k$ for $m$ large enough and $z \notin J$, that $G^{(1)}(z)$ is analytic for $z \notin J$ except at the zeros of $Q\left(T^{i}(z)\right), i=0,1,2, \ldots$, that do not lie in $J$. Since $G^{(1)}(z)$ is a Stieltjes function all its isolated singularities are simple poles and the theorem now follows because each zero of $Q\left(T^{i}(z)\right)$ that is not in $J$ is not an accumulation point of other zeros of $Q\left(T^{j} z\right), j=0,1,2, \ldots$, and is therefore isolated.

Remark. 1. Denoting $P_{n}, P_{n}^{(1)}$ and $P_{n}^{(2)}$ as the monic orthogonal polynomials of degree $n$ associated with $\mathscr{J}, \mathscr{J}^{(1)}$ and $\mathscr{J}^{(2)}$ (omit first two rows and columns of $\mathscr{J}$ ) respectively one finds that

$$
P_{N^{n}}=T^{n}(z)-b(0), \quad P_{N^{n-1}}^{(1)}=\prod_{i=1}^{n-1} Q\left(T^{n} z\right) \quad \text { and } \quad P_{N-2}^{(2)}=R(z) .
$$

Labelling the zeros of $Q(z)$ by $z_{1}, z_{2}, \ldots, z_{N-1}$, it follows that the zeros of $Q\left(T^{j}(z)\right)$ are $z_{k, m}^{(j)}=T_{k}^{-j}\left(z_{m}\right)$ with $m=1,2, \ldots, N-1$ and $k=1,2, \ldots, N^{j}$. Here $\left\{T_{k}^{-j}, k=1,2, \ldots, N^{j}\right\}$ denotes a complete assignment of branches of $T^{-j}$. Supposing that $z_{k, m}^{(j)} \notin J$ one finds that the residue of $G^{(1)}(z)$ at that point is

$$
\Gamma_{k, m}^{(j)}=\sum_{l=j}^{\infty} \frac{R\left(T^{l}\left(z_{k, m}^{(j)}\right)\right)}{Q^{\prime}\left(T^{j} z_{k, m}^{(j)}\right) \prod_{\substack{i=0 \\ i \neq j}}^{l} Q\left(T^{i} z_{k, m}^{(j)}\right)} .
$$

Using the fact that $z_{k, m}^{(j)}$ is one of the $j^{\text {th }}$ inverse iterates of $z_{m}$, and using (II.4) and (II.11) yields

$$
\Gamma_{k, m}^{(j)}=\frac{-P_{N}\left(z_{m}\right) \Gamma_{m}}{a(1) P_{N^{j+1}-1}^{(1)}\left(z_{k, m}^{(j)}\right)}
$$

where

$$
\Gamma_{m}=1-a(1) \sum_{l=1}^{\infty} \frac{R\left(T^{l} z_{m}\right)}{P_{N}\left(z_{m}\right)} \prod_{s=1}^{l} 1 / Q\left(T^{s} z_{m}\right)
$$


Lemma 1. Let $I$ be the smallest real interval containing $J$ and suppose $I=[-a, a]$. Let $z_{m}$ be such that $Q\left(z_{m}\right)=0$ and $z_{m} \notin J$, then

$$
\left|\prod_{i=2}^{l-1} Q\left(T^{i} a_{m}\right)\right| \geqq\left|Q\left(T^{2} z_{m}\right)\right|^{l-2}, \quad l=3,4, \ldots,
$$

and

$$
\left|\frac{R\left(T^{l} z_{m}\right)}{Q\left(T^{l} z_{m}\right)}\right| \leqq\left|\frac{R\left(T^{2} z_{m}\right)}{Q\left(T^{2} z_{m}\right)}\right|, \quad l=2,3, \ldots .
$$

Proof. Since $I$ is the smallest interval containing the Julia set, one has that $T z$ is expanding for $|z|>a$. Furthermore for $z$ real $\notin I, T^{l} z, l \geqq 1$ is of fixed sign. Since the zeros of $Q(z)$ lie at the finite maxima and minima of $T z$, the assumptions that $J$ is real and that $z_{m} \notin J$ imply that $\left|T z_{m}\right|>a$. The fact that the zeros of $Q(z)$ interlace those of $T(z)$ imply that $Q(z)$ is monotonically increasing for $z \geqq a$ and $|Q(z)|$ is monotonically decreasing for $z \leqq-a$. Consequently $\left|Q\left(T^{l} z_{m}\right)\right| \geqq$ $\left|Q\left(T^{2} z_{m}\right)\right|$, for $l \geqq 2$ which yields (II.15). (II.16) follows from the above arguments and the fact that $R(z) / Q(z)$ is an $[N-2 / N-1]$ Padé approximant (see (II.11)) with positive residues, and with all its zeros and poles strictly inside $I$.

Lemma 2. Let $I, J$ and $z_{m}$ be as in Lemma 1. If $T z_{m}>0$ then

$$
\begin{gathered}
\left|Q\left(T^{2} z_{m}\right)\right|>\left|Q\left(T z_{m}\right)\right|, \\
\left|\frac{R\left(T^{2} z_{m}\right)}{Q\left(T^{2} z_{m}\right)}\right|<\left|\frac{R\left(T z_{m}\right)}{Q\left(T z_{m}\right)}\right|<\left|\frac{R(a)}{Q(z)}\right|,
\end{gathered}
$$

and

$$
\left|P_{N}\left(z_{m}\right)\right|=\left|T z_{m}-b(0)\right| \geqq|a-b(0)| .
$$

If $T z_{m}<0$, and $T a=a$ and $T(-a)=-a$, then (II.17) remains unchanged, and (II.18) and (II.19) remain valid with $a \rightarrow-a$.

Proof. Inequalities (II.17) and (II.18) follow from the same argument given in Lemma 1 and the fact that $T^{2} z_{m}$ has the same sign as $T z_{m}$. Equation (II.19) follows from the expanding nature of $T$ on $I^{c}$ and the fact that $-a<b(0)=\int_{J} x d u<a$.

Theorem 2. Let $J$ be real and $z_{m} \notin J$, then $\Gamma_{m}>0$.

Proof. Let $I$ be the smallest real interval containing $J$. It is without loss of generality that $I=[-a, a]$. If this is not the case, by using a mobius transform of the form $L z=z+c, c$ real we can symmetrize $J$ without changing either the monic character of $T$, or the nature of the fixed points of $T$, or the fact that $z_{m} \notin J$. Since $I$ is the smallest interval containing $J$, one has that $T a=a$ and either $T(-a)=a$ or $T(-a)=-a$ depending upon whether the degree of $T$ is even or odd. The proof now breaks up into two cases; Case $1, T z_{m}>0$ or $T z_{m}<0$ and $T(-a)=-a$, and Case 2, $T z_{m}<0$ and $T(-a)=a$. To prove Case 1 we note that a consequence of (II.15) and (II.16) of Lemma 1 and, (II.17) and (II.18) of Lemma 2 in (II.14) is

$$
\Gamma_{m}>1-\left|a(1) \frac{R( \pm a)}{P_{N}( \pm a) Q( \pm a)}\right| \sum_{i=0}^{\infty} \frac{1}{\left|Q\left(T z_{m}\right)\right|^{i}} .
$$


Here one chooses $a$ if $T z_{m}>0$ and $-a$ if $T z_{m}<0$. From the expanding nature of the $T$ on $I^{c}$, the monotonicity properties of $Q$ on $I^{c}$, and (II.4) one finds that

$$
\left|Q\left(T z_{m}\right)\right|>|Q(T \pm a)|=\left|1+\frac{R( \pm a) a(1)}{( \pm a-b(0))}\right|>1 \text {. }
$$

The last inequality follows from the fact that $R( \pm a)=( \pm a-b(0) / a(1))$. Since $R( \pm a) / \pm a-b(0)$ and $Q( \pm a)$ are positive it is a consequence of the above equation and (II.4) that

$$
\Gamma_{m}>1-\frac{1-1 / Q( \pm a)}{1-1 / Q\left(T z_{m}\right)}>0
$$

where again $a$ is chosen if $T z_{m}>0$ and $-a$ is chosen if $T z_{m}<0$.

To prove Case 2 we note that in this case $Q\left(T^{i} z_{m}\right)>0, i \geqq 2, R\left(T^{i} z_{m}\right)>0, i \geqq 2$, $Q\left(T z_{m}\right)<0$ and $P_{N}\left(z_{m}\right)<0$. Therefore (II.14) becomes using Lemma 1,

$$
\Gamma_{m}>1-\frac{a(1) R\left(T z_{m}\right)}{P_{N}\left(z_{m}\right) Q\left(T z_{m}\right)}-\frac{a(1) R\left(T^{2} z_{m}\right)}{P_{N}\left(z_{m}\right) Q\left(T z_{m}\right)\left(Q\left(T^{2} z_{m}\right)-1\right)} .
$$

Here we have used the fact that $Q\left(T^{2} z_{m}\right)>1$. Now using (II.4) yields

$$
\Gamma_{m}>1-\left(\frac{P_{N}\left(T z_{m}\right) Q\left(T z_{m}\right)-P_{N}\left(T z_{m}\right)}{P_{N}\left(z_{m}\right) Q\left(T z_{m}\right)}\right)-\left(\frac{\left(P\left(T z_{m}\right) Q\left(T^{2} z_{m}\right)-P\left(T^{2} z_{m}\right)\right)}{P_{N}\left(z_{m}\right) Q\left(T z_{m}\right)\left(Q\left(T^{2} z_{m}\right)-1\right)}\right),
$$

which yields

$$
\Gamma_{m}>\frac{P\left(T^{2} z_{m}\right)-P\left(T z_{m}\right)}{P_{N}\left(z_{m}\right) Q\left(T z_{m}\right)\left(Q\left(T^{2} z_{m}\right)-1\right)}>0 .
$$

The above result has the following immediate consequences.

Corollary 1. Let $J$ be real and suppose at least one zero of $Q(z)$ lies outside $J$, then $\mathscr{J}^{(1)}$ has an infinite number of eigenvalues.

Proof. From Theorem 2 we have that $\Gamma_{m}>0$. The result now follows from (II.13) and the fact that the zeros of $P_{N}(z)$ and $P_{N}^{(1)+1}-1(z)$ are simple.

The following corollary is a consequence of Theorem 2 and the fact that

$$
\sum_{j=0}^{\infty} \sum_{k=1}^{N J} \Gamma_{k, m}^{(j)}<1
$$

Corollary 2. For every $\varepsilon>0$ there exists $a \gamma$ and $w$ such that

$$
\begin{gathered}
\sum_{j=\gamma}^{\infty} \sum_{k=1}^{N^{j}}\left|\frac{1}{P_{N^{j+1}-1}^{(1)}\left(z_{k, m}^{(j)}\right)^{\prime}}\right|<\varepsilon, \\
\sum_{l=w}^{\infty}\left|\frac{R\left(T^{l} z_{m}\right)}{P_{N}\left(z_{m}\right)} \prod_{s=1}^{l} \frac{1}{Q\left(T^{s} z_{m}\right)}\right|<\varepsilon .
\end{gathered}
$$

We now show how dramatically the spectrum may change in going from $\mathscr{J}$ to $\mathscr{J}^{(1)}$.

Theorem 3. Let $J$ be real and let all the zeros of $Q(z)$ lie outside $J$, then the spectrum of $\mathscr{J}^{(1)}$ is purely discrete. 
Proof. Since $\int_{-\infty}^{\infty} d u^{(1)}=1$ we will prove the result by showing that the sum of all the residues of $G^{(1)}(z)$ is equal to 1. From (II.11) one sees that the first term in (II.3) is just the $[N-2 / N-1]$ Padé approximant to $G^{(1)}(z)$.

Now consider the sum of the first two terms in (II.3)

$$
\begin{aligned}
\frac{R(T z)}{Q(z) Q(T z)}+\frac{R(z)}{Q(z)} & =\frac{1}{a(1)}\left[\frac{P_{N}(z) Q(T z)-P_{N}(T z)}{Q(z) Q(T z)}+\frac{(z-b(0)) Q(z)-P_{N}(z)}{Q(z)}\right] \\
& =\frac{1}{a(1)^{2}}\left[\frac{(z-b(0)) Q(z) Q(T z)-P_{N}(T z)}{Q(z) Q(T z)}\right] \\
& =\frac{P_{N-2}^{(2)}(z)}{P_{N-1}^{(1)}(z)}
\end{aligned}
$$

which is just the $\left[N^{2}-2 / N^{2}-1\right]$ Padé approximant to $G^{(1)}(z)$. Proceeding by induction it is not hard to see that the sum of the first in terms in (II.3) give the $\left[N^{n}-2 / N^{n}-1\right]$ Padé approximant to $G^{(1)}(z)$. Labelling the residues of $\left[\left(N^{n}-2\right) /\right.$ $\left.N^{n}-1\right]$ by ${ }^{n} \Gamma_{k, m}^{(j)}$, where $j, k$ and $m$ have the same meanings as in (II.12), one finds that

$$
\sum_{j=0}^{n-1} \sum_{k=1}^{N J} \sum_{m=1}^{N-1}{ }^{n} \Gamma_{k, m}^{(j)}=1
$$

Subtracting ${ }^{n} \Gamma_{k, m}^{(j)}$ from $\Gamma_{k, m}^{(j)}$ and using (II.14) yields,

$$
\Gamma_{k, m}^{(j)}-{ }^{n} \Gamma_{k, m}^{(j)}= \begin{cases}\Gamma_{k, m}^{(j)} & j \geqq n \\ \frac{1}{P_{N^{j+1}-1}^{(1)}\left(z_{k, m}^{(j)}\right)^{\prime}} \sum_{l=n-j}^{\infty} R\left(T^{l} z_{m}\right) \prod_{s=1}^{l} \frac{1}{Q\left(T^{s} z_{m}\right)}, & j<n .\end{cases}
$$

Set $M=\max (A, B)$, where $A=\max _{m}\left|P_{N}\left(z_{m}\right) \Gamma_{m}\right|$ and

$$
B=\max _{m} \sum_{l=1}^{\infty}\left|R\left(T^{l} z_{m}\right) \prod_{i=1}^{l} \frac{1}{Q\left(T^{l} z_{m}\right)}\right|,
$$

then Corollary 2 implies that there exists a $\gamma$ such that

$$
\left.M \sum_{m=1}^{N-1} \text { L.H.S. (II. } 21\right)<\varepsilon / 2 \text {. }
$$

From the definition of $P_{N^{j+1}-1}^{(1)}(z)$, (II.11), we see that

$$
P_{N^{j+1}-1}^{(1)}\left(z_{k, m}^{(j)}\right)^{\prime}=N^{j} Q^{\prime}\left(z_{m}\right) P_{N^{j}-1}\left(z_{k, m}^{j}\right)^{2} .
$$

Setting

$$
M_{1}=\max _{j \leqq \gamma, k \leqq N^{j}, m}\left|\frac{1}{Q^{\prime}\left(z_{m}\right) P_{N^{j-1}}\left(z_{k, m}^{j}\right)^{2}}\right|,
$$

it is a consequence of Corollary 2 that there exists an $w$ large enough such that

$$
M_{1} \gamma \sum_{m=1}^{N-1} \text { L.H.S. (II.22) }<\varepsilon / 2 .
$$


Now choosing $n$ large enough in (II.24) so that $n-\gamma>w$, we have from the above arguments that

$$
\begin{aligned}
& \left|\sum_{m=1}^{N-1} \sum_{j=0}^{\infty} \sum_{k=0}^{N^{j}}\left(\Gamma_{k, m}^{(j)}-{ }^{n} \Gamma_{k, m}^{(j)}\right)\right| \\
& =\left|\sum_{m=1}^{N-1} \sum_{j=0}^{\infty} \sum_{k=1}^{N^{j}} \Gamma_{k, m}^{(j)}-1\right| \\
& <M \sum_{m=1}^{N-1} \sum_{j=\gamma}^{\infty} \sum_{k=1}^{N^{j}}\left|\frac{1}{P_{N^{j+1}}^{(1)}\left(z_{k, m}^{(j)}\right)}\right| \\
& \quad+M_{1} \sum_{m=1}^{N-1} \sum_{j=0}^{\gamma-1} \sum_{k=1}^{N^{j}} \frac{1}{N^{j}} \sum_{l=n-j}^{\infty}\left|R\left(T^{l} z_{m}\right) \prod_{s=1}^{l} \frac{1}{Q\left(T^{i} z_{m}\right)}\right|<\varepsilon,
\end{aligned}
$$

thus yielding the result.

\section{Limit Periodic Behavior}

In Sect. II we make frequent reference to the following result, which is proved in [17] (Theorem 3 there).

Orthogonality Theorem. If $f \in L^{1}(J, \mu)$, then

$$
\int_{J} P_{l}(z) f\left(T^{n}(z)\right) d \mu(z)=0, \quad l, m \in \mathbb{N}_{0},
$$

whenever $N^{n}$ does not divide $l$.

We also make use of [4, Theorem 2].

Reduction Theorem. If $f \in L^{1}(J, \mu)$, then

$$
\int_{J} z^{j} f(T(z)) d \mu(z)=\frac{s_{j}}{N} \int f(z) d \mu(z), \quad j=1,2, \ldots, N-1,
$$

where the $s_{j}$ 's are given recursively by

$$
s_{m}=-m k_{m}-\sum_{l=1}^{m-1} k_{l} s_{m-l}, \quad m=1,2,3, \ldots .
$$

Throughout we suppose that $J \subset \mathbb{R}$ and that $T(z)$ is a polynomial of degree $N \geqq 2$. Then it is well known that the monic orthogonal polynomials associated with $\mu$ satisfy the recurrence formula

$$
P_{n+1}(x)+b(n) P_{n}(x)+a(n) P_{n-1}(x)=x P_{n}(x), \quad n \in \mathbb{N}_{0}, \quad P_{-1}(x)=0, \quad P_{0}(x)=1,
$$

where the $a(n)$ 's and $b(n)$ 's are defined in (I.5) and (I.6). We will always understand that $b(n-1)=a(n)=0$ and $P_{n-1}(x)=0$ whenever $n \leqq 0$. Also throughout we use the notation $\mathbb{N}=\{1,2,3, \ldots\}$ and $\mathbb{N}_{0}=\mathbb{N} \cup\{0\}$; and the value of a summation where the lower index exceeds the upper index is zero. $T(z)$ has definite parity when $T(z) \equiv T(-z)$ or $T(z) \equiv-T(-z)$.

Lemma 3. Let $T(z)=z^{N}+k_{2} z^{N-2}+\cdots+k_{N}$ be of definite parity, then the 
following relations hold for all $n \in N_{0}$ :

and

$$
\begin{gathered}
a(n N) a(n N-1) \ldots a(n N-N+1)=a(n), \\
a(n N)+a(n N+1)=-2 k_{2} / N, \\
b(n)=0,
\end{gathered}
$$

$$
\begin{aligned}
a(n N+m)= & \frac{\left\langle P_{m+1} P_{n N+m-1} P_{n N}\right\rangle}{\left\langle P_{n N+m-1}^{2}\right\rangle} \\
& +\sum_{l=1}^{m}(a(l)-a(n N+m-l)), \quad m \in N
\end{aligned}
$$

Proof. Equation (III.2) was derived in [8]. To prove (III.3) we begin with

$$
a(n N+1)=\frac{\left\langle P_{n N+1}^{2}\right\rangle}{\left\langle P_{n N}^{2}\right\rangle}=\frac{\left\langle x^{2} P_{n N}^{2}\right\rangle}{\left\langle P_{n N}^{2}\right\rangle}-a(n N), \text { for } n \in \mathbb{N}_{0},
$$

where (III.1) has been twice. The result now follows upon employing the Reduction Theorem.

Equation (III.4) follows from the fact that if $T(z)$ is of definite parity then $J$ is symmetric with respect to the origin and $\mu$ is invariant under change of sign. Consequently, all of the $P_{n}(x)$ 's have definite parity and (I.6) shows that $b(n)=0$.

To show (III.5) we begin with

$$
a(n N+m)=\frac{\left\langle P_{n N+m}^{2}\right\rangle}{\left\langle P_{n N+m-1}^{2}\right\rangle}, \text { for } n \in \mathbb{N}_{0}, \quad m \in \mathbb{N} .
$$

Using the recurrence formula twice yields

$$
a(n N+m)=\frac{\left\langle x^{2} P_{n N+m-1}^{2}\right\rangle}{\left\langle P_{n N+m-1}^{2}\right\rangle}-a(n N+m-1) .
$$

which can be written

$$
a(n N+m)=\frac{\left\langle P_{2} P_{n N+m-1}^{2}\right\rangle}{\left\langle P_{n N+m-1}^{2}\right\rangle}+a(1)-a(n N+m-1) .
$$

Now eliminate one of the $P_{n N+m-1}$ 's using (III.1) and then eliminate $x P_{2}$ using (III.1) to obtain

$$
a(n N+m)=\frac{\left\langle P_{3} P_{n N+m-1} P_{n N+m-2}\right\rangle}{\left\langle P_{n N+m-1}^{2}\right\rangle}+\sum_{j=1}^{2}(a(j)-a(n N+m-j)) .
$$

Continuing this procedure yields (III.5).

Corollary 3. If $T(z)$ is of definite parity then for $n \in \mathbb{N}_{0}$,

$$
a(n N+N-1)=\sum_{j=1}^{N-1}(a(j)-a(n N+N-j-1)) .
$$

That is, $a(0+n N)+a(1+n N)+\cdots+a(n-1+n N)$ is independent of $n \in \mathbb{N}_{0}$.

Proof. This follows from (III.5) and the Orthogonality Theorem. 
Lemma 4. Suppose $T(z)$ is of definite parity and that $a(j N+i)=\alpha^{2}$ for $i=2,3, \ldots$, $N-1$ and $j=0,1, \ldots,(n-1)$, for some $n \in \mathbb{N}$, then

$$
\begin{aligned}
\left\langle P_{m} P_{j N+k} P_{j N}\right\rangle=0 \text { for }|k| & =0,1, \ldots, N-1 \text { with }|k| \neq m, \\
m & =0,1, \ldots, N, \\
j & =0,1, \ldots, n .
\end{aligned}
$$

Proof. We note that $\left(^{*}\right)$ is immediately true in the following cases: whenever $m<|k|$, by orthogonality of the $P_{n}$ 's; whenever $j=0$; whenever $m=N$ and $|k| \neq 0$ by the Orthogonality Theorem; and whenever $m=N$ and $k=0$, by parity (which implies all of the $b$ 's vanish). Thus, let us assume $\left(^{*}\right)$ is true whenever $j=0,1, \ldots,(n-1)$; then we will show that it is true when $j=n$. Here we assume $m<N$ since the case $m=N$ has already been taken care of. Choosing $k=-1$, we use the recurrence formula (III.1) to obtain, for $m<N$,

$$
\begin{aligned}
\left\langle P_{m} P_{n N-1} P_{n N}\right\rangle= & \left\langle x P_{m-1} P_{n N-1} P_{n N}\right\rangle-a(m-1)\left\langle P_{m-2} P_{n N-1} P_{n N}\right\rangle \\
= & \left\langle P_{m-1} P_{n N}^{2}\right\rangle+a(n N-1)\left\langle P_{m-1} P_{n N-2} P_{n N}\right\rangle \\
& -a(m-1)\left\langle P_{m-2} P_{n N-1} P_{n N}\right\rangle .
\end{aligned}
$$

The first term is equal to zero by the Orthogonality Theorem. If $m=2$ the remaining two terms are equal to zero by orthogonality. If $m$ is greater than 2 then one eliminates $P_{n N-1}$, and then $x P_{m-2}$ in the last equation, with the aid of (III.1), which yields

$$
\begin{aligned}
\left\langle P_{m} P_{n N-1} P_{n N}\right\rangle= & (a(n N-1)-a(m-1))\left\langle P_{m-1} P_{n N-2} P_{n N}\right\rangle \\
& +a(m-1)\left[a(n N-2)\left\langle P_{m-2} P_{n N-3} P_{n N}\right\rangle\right. \\
& \left.-a(m-2)\left\langle P_{m-3} P_{n N-2} P_{n N}\right\rangle\right] .
\end{aligned}
$$

The first term here vanishes by the supposition in the statement of the lemma. The coefficient of $a(m-1)$ is zero when $m<5$ by orthogonality. If $m \geqq 5$ we repeat the procedure until a proof which works for $m<N$ is arrived at.

Next we carry out an induction through negative values of $k$. We use the identity

$$
\begin{aligned}
\left\langle P_{m} P_{n N-l} P_{n N}\right\rangle= & \frac{1}{a(n N-l+1)}\left[\left\langle P_{m+1} P_{n N-l+1} P_{n N}\right\rangle\right. \\
& \left.+a(m)\left\langle P_{m-1} P_{n N-l+1} P_{n N}\right\rangle-\left\langle P_{m} P_{n N-l+2} P_{n N}\right\rangle\right] .
\end{aligned}
$$

(*) now follows for $k<0$ by induction and the fact that it is true for $m=N$.

To obtain $(*)$ with $j=n$ when $k \geqq 0$ observe the identity

$$
\begin{aligned}
\left\langle P_{m} P_{n N+k} P_{n N}\right\rangle= & \left\langle x P_{m} P_{n N+k-1} P_{n N}\right\rangle-a(n N+k-1)\left\langle P_{m} P_{n N+k-2} P_{n N}\right\rangle \\
= & \left\langle P_{m+1} P_{n N+k-1} P_{n N}\right\rangle-a(m)\left\langle P_{m-1} P_{n N+k-1} P_{n N}\right\rangle \\
& -a(n N+k-1)\left\langle P_{m} P_{n N+k-2} P_{n N}\right\rangle,
\end{aligned}
$$

which allows us to carry out an induction through positive values of $k$, upon recalling the observations at the beginning of the proof.

We are now in a position to consider the coefficients associated with the scaled Chebychev polynomials. 
Theorem 4. If $T(z)=\alpha^{N} C_{N}(z / \alpha)$ with $N \geqq 2$ and $\alpha \neq 0$, where $C_{N}(z)$ is the degree $N$ monic Chebychev polynomial of the first kind, orthogonal on $[-2,2]$, then the following relations hold for all $n \in \mathbb{N}_{0}$,

$$
\begin{aligned}
& a(n N) a(n N-1) \ldots a(n N-N+1)=a(n), \\
& \quad a(n N+1)+a(n N)=2 \alpha^{2}, \\
& a(n N+1)=\alpha^{2}, \text { for } j=2,3, \ldots, N-1 .
\end{aligned}
$$

Proof. Equations (III.7) and (III.8) follow at once from Theorem 1. To prove (III.9) begin by noting that $T_{N}(z)$ is the monic Chebychev polynomial of order $N$ on the interval $[-2 \alpha, 2 \alpha]$. As such one has by moment analysis $[17,20]$ that the first $N$ monic orthogonal polynomials $\left\{P_{m}\right\}_{m=0}^{N-1}$ associated with the invariant measure for $T(z)$ on the Julia set are the same as the first $N$ scaled Chebychev polynomials; namely $P_{m}(z)=\alpha^{m} C_{m}(z / \alpha)$ for $m=0,1,2, \ldots, N-1$. Therefore from the standard formulas for Chebychev polynomials [20] one has $a(l)=\alpha^{2}$ for $l=2,3, \ldots, N-1$. The result now follows from induction using Lemma 4 and (III.5)

Remark 2. It is interesting to note that in these cases for large $N$ the Jacobi matrices have a large proportion of constant terms. However, the spectrum for all finite $N$ and $\alpha>1$ is a Cantor set.

Definition 1 [20]. A bounded sequence $\{b(n)\}_{n=-\infty}^{\infty}$ is called limit periodic if it is the uniform limit of bounded periodic sequences; that is, there exist periodic sequences $\left\{b^{(m)}(n)\right\}_{n=-\infty}^{\infty}$ such that

$$
\left.\lim _{m \rightarrow \infty}\left(\sup _{n}\left|b(n)-b^{(m)}(n)\right|\right)\right)=0 .
$$

(A sequence $\left\{b^{(m)}(n)\right\}_{-\infty}^{\infty}$ is called periodic when and only when there exists a positive integer $L_{m}$ such that

$$
b^{(m)}\left(n+L_{m}\right)=b^{(m)}(n) \text { for all } n=0, \pm 1, \pm 2, \ldots
$$

Theorem 5. Let $T(z)=\alpha^{N} C_{N}(z / \alpha)$ where $N \geqq 2$ and $\alpha>1$. Then

$$
\operatorname{Lim}_{n \rightarrow \infty} a\left(m N^{n}+s\right)=a(s) \text { for all } s \in \mathbb{N}_{0}, \quad m \in \mathbb{N}_{0} .
$$

\section{Moreover}

$\left|a\left(m N^{n}+s\right)-a(s)\right| \leqq 2 \alpha^{2} \gamma^{n}$ for all $s \in \mathbb{N}_{0}, n \in \mathbb{N}, m \in \mathbb{N}$, where $\gamma=1 /\left\{\left(2 \alpha^{2}-2\right) \cdot \alpha^{2(N-2)}\right\}$. In particular $\{a(n)\}_{n=-\infty}^{\infty}$ can be redefined for $n<0$ so that it form a limit periodic sequence whenever $\left(2 \alpha^{2}-2\right) \cdot \alpha^{2(N-2)}>1$ (which is always true when $\left.\alpha>\sqrt{(3 / 2)}\right)$.

Proof. The arguments used for proving the first assertion are basically the same as those given in [22] and [9], and will only be sketched here. Both results are immediate if $s=k \bmod N, k=2,3, \ldots, N-1$, since all of the corresponding $a$ 's are equal to $\alpha^{2}$. For $s=0 \bmod N$ we observe from Theorem 2 that

$$
a(m N)=\alpha^{-2(N-2)} \cdot \frac{a(m)}{2 \alpha^{2}-a((m-1) N)} .
$$

Using this it follows by induction that for $\alpha>1$ we have $0<a(n N)<a(n)$. Therefore 


$$
a\left(m N^{n}\right)<\frac{\alpha^{-2(N-2) n} a(m)}{\left(2 \alpha^{2}-1\right)^{n}}<\frac{2 \alpha^{-2((N-2) n-1)}}{\left(2 \alpha^{2}-1\right)^{n}}
$$

for $n \in \mathbb{N}$, where we have used $a(n) \leqq 2 \alpha^{2}$. Thus

$$
\operatorname{Lim}_{n \rightarrow \infty} a\left(m N^{n}\right)=0=a(0) .
$$

The rest of the assertions in the first part of the theorem now follow by repeated application of Theorem 4 .

To prove the second part of the theorem, we first note that from the above inequality it is true for $s=0$, and $s=k \bmod N$, for $k=2,3, \ldots, N-1$. The case $s=$ $1 \bmod N$ can be reduced to the case $s=0 \bmod N$ using $\left|a\left(m N^{n}+1\right)-a(1)\right|=$ $a\left(m N^{n}\right)$. Therefore we consider

$$
a\left(m N^{n}+N s\right)-a(N s)=\frac{a\left(m N^{n-1}+s\right)-a(s)+a(s)-a(N s) \beta a\left(m N^{n}+N s-N+1\right)}{\beta a\left(m N^{n}+N s-N+1\right)},
$$

where $\beta=\alpha^{2(N-2)}$ and we have used (III. 7). Using (III. 8) this can be rewritten

$$
\begin{aligned}
a\left(m N^{n}+N s\right)-a(N s)= & \frac{a\left(m N^{n-1}+s\right)-a(s)}{\beta\left(2 \alpha^{2}-a\left(m N^{n}+N s-N\right)\right)} \\
& +\frac{a(N s)\left(a(N s-N+1)-a\left(m N^{n}+N s-N+1\right)\right)}{2 \alpha^{2}-a\left(m N^{n}+N s-N\right)} .
\end{aligned}
$$

Therefore, assuming $\left|a\left(m N^{n}+j\right)-a(j)\right| \leqq 2 \alpha^{2} \gamma^{n}$ whenever $j<N s$, we have

$$
\begin{aligned}
\left|a\left(m N^{n}+N s\right)-a(N s)\right| \leqq & \frac{2 \alpha^{2} \cdot\left(2 \alpha^{2}-2\right)^{-n+1}}{\beta^{n} \cdot\left(2 \alpha^{2}-1\right)} \\
& +\frac{2 \alpha^{2} \cdot\left(2 \alpha^{2}-2\right)^{-n}}{\beta^{n} \cdot\left(2 \alpha^{2}-1\right)}=2 \alpha^{2} \gamma^{n},
\end{aligned}
$$

as desired, where the fact that $a(N s)<1$ and $|a(i)-a(j)|<2 \alpha^{2}$ for all $i$ and $j$ has been used.

We now prove the limit periodicity assertion. We assume $\gamma<1$ and redefine $a(s)$ for $s<0$ by

$$
a(s)=\operatorname{Lim}_{k \rightarrow \infty} a\left(m N^{k}+s\right) .
$$

The limit exists for each $m$ because $a\left(m N^{k}+s\right)$ is a Cauchy sequence in $k$, as the following computation shows. We have for $\tilde{k}>k$ and $m N^{k}+s>0$,

$$
\begin{aligned}
\left|a\left(m N^{\tilde{k}+s}+s\right)-a\left(m N^{k}+s\right)\right| & =\left|a\left(m N^{\tilde{k}}-m N^{k}+m N^{k}+s\right)-a\left(m N^{k}+s\right)\right| \\
& =\left|a\left(\left(m N^{\tilde{k}-k}-1\right) N^{k}+m N^{k}+s\right)-a\left(m N^{k}+s\right)\right| \\
& \leqq 2 \alpha^{2} \gamma^{k}
\end{aligned}
$$

The limit is independent of $m$ because

$$
\left|a\left(m N^{k}+s\right)-a\left(\tilde{m} N^{k}+s\right)\right|=\left|a\left((m-\tilde{m}) N^{k}+\tilde{m} N^{k}+s\right)-a\left(\tilde{m} N^{k}+s\right)\right| \leqq 2 \alpha^{2} \gamma^{k}
$$


whenever $k$ is sufficiently large. Furthermore, we now have

$$
\left|a\left(m N^{k}+s\right)-a(s)\right|=\operatorname{Lim}_{\tilde{k} \rightarrow \infty}\left|a\left(m N^{k}+m N^{\widetilde{k}}+s\right)-a\left(m N^{\widetilde{k}}+s\right)\right| \leqq 2 \alpha^{2} \gamma^{k} .
$$

We have shown that when $\gamma<1$ we can redefine $a(n)$ for $n<0$ so that

$$
\left|a\left(m N^{n}+s\right)-a(s)\right| \leqq 2 \alpha^{2} \gamma^{n} \text { for all } s \in \mathbb{Z}, n \in \mathbb{N}, m \in \mathbb{N} .
$$

We now define sequences $\left\{a^{\left(N^{k}\right)}(n)\right\}_{n=-\infty}^{\infty}$ for $k=1,2,3, \ldots$ by $a^{\left(N^{k}\right)}(n)=a\left(n \bmod N^{k}\right)$, from which it follows at once that

$$
\sup _{n}\left|a^{\left(N^{k}\right)}(n)-a(n)\right| \leqq 2 \alpha^{2} \gamma^{k}
$$

and

$$
\operatorname{Lim}_{k \rightarrow \infty} \sup _{n}\left|a^{\left(N^{k}\right)}(n)-a(n)\right|=0 .
$$

The following result is immediate.

Corollary 4. when $\gamma<1$ the frequency module of $a=\{a(n)\}$ is contained in the set of all numbers for the form $2 \pi(N p+k) / N^{n}$ where $n \geqq 1,0 \leqq p \leqq N^{n-1}, 1 \leqq k \leqq N-1$. That is, we have Fourier representations of the form

$$
a(n)=\Gamma_{0,0,0}+\sum_{n=1}^{\infty} \sum_{p=0}^{N n-1} \sum_{k=1}^{N-1} \Gamma_{n, p, k} \exp \left\{2 \pi i(N p+k) / N^{n}\right\}
$$

We define

$$
\mathscr{J}^{(n)}=\left(\begin{array}{ccc}
0 & c(n+1) & 0 \\
c(n+1) & 0 & c(n+2) \cdot \\
0 & c(n+2) & 0 \cdot \\
0 & 0 &
\end{array}\right) \text { for } n \in \mathbb{N}_{0}
$$

where we recall $c(n)^{2}=a(n)$ and $c(n)>0$ when $J \subset \mathbb{R}$. Then $\mathscr{J}^{(n)}$, being bounded, can be considered as a self-adjoint operator on $l_{2}^{+}$, and Theorem 5 provides the following result.

Corollary 5. If $\gamma>1$ then $\mathscr{J}^{\left(m N^{p+s)}\right.} \rightarrow \mathscr{J}^{(s)}$ as $p \rightarrow \infty$ for fixed $s \in \mathbb{N}_{0}, m \in \mathbb{N}_{0}$, the convergence taking place in the strong operator topology on $B\left(l_{2}^{+}\right)$. If $\gamma<1$, which is always true when $\alpha>\sqrt{(3 / 2)}$, then the convergence is in the norm topology on $B\left(l_{2}^{+}\right)$. (The topologies here are defined by Reed and Simon [23].)

Remark 3. If $\alpha=1$, then $a(1)=2$ and $a(n)=1$ for $n \geqq 2$, whence the above result holds for $s \in \mathbb{N}$ but not for $s=0$.

Remark 4. Using the $a(n)$ 's redefined for negative $n$ as in Theorem 5, and correspondingly defining $\bar{c}=\{c(n)\}_{n=-\infty}^{\infty}$ and $\bar{c}_{m}=\{c(n+m)\}_{n=-\infty}^{\infty}$ for $m \in \mathbb{Z}$, define a transformation $T_{n}$ by $T_{n} \bar{c}_{m}=\bar{c}_{m}=\bar{c}_{m+n}$. Extending $\mathscr{J}^{(n)}$ to a doubly infinite matrix $\mathscr{J}\left(\bar{c}_{n}\right) \in B\left(l_{2}\right)$, we see that $T_{n}$ has a realization in $B\left(l_{2}\right)$ [13] of the form $T_{n} \mathscr{J}\left(\bar{c}_{m}\right)=$ $\mathscr{J}\left(T_{n} \bar{c}_{m}\right)=S^{-n} \mathscr{J}\left(\bar{c}_{m}\right) S^{n}$, where $S$ is the unitary matrix which obeys $(S \psi)(n)=\psi(n-1)$, 
and $S^{n}=S \cdot S^{n-1}$. Theorem 3 says that for $\gamma<1, T$ can be viewed as a continuous limit periodic flow [24] on the hull of $\mathscr{J}(\bar{c})$.

Remark 5. We have also considered the transformations $T z=\alpha^{3} C_{3}(z / \alpha)+B$ and $T z=\gamma^{4} C_{4}(z / \gamma)+\Delta$ and found that for $\alpha \geqq 5$ and $|B| \leqq 5$, and $\gamma \geqq 2$ and $|\Delta|<22$ the coefficients in the Jacobi matrices associated with the above two transformations are limit periodic [25].

\section{Physical Consequences}

The above results have a number of physical consequences. Here we shall for simplicity consider the transformation $T z=z^{2}-2 \alpha$. Writing

$$
G(E)=\left\langle 0\left|(E-\mathscr{J})^{-1}\right| 0\right\rangle=\left(E-a(1) G^{(1)}(E)\right)^{-1},
$$

where in the language of solid state physics [26-29] $G(E)$ is the $\langle 0|| 0$,$\rangle matrix$ element of the Green's function and $G^{(1)}(E)$ is the "self-energy", one sometimes determines numerically whether $G(E)$ has extended states by determining whether or not $G^{(1)}(E)$ has a branch cut. In the above model with $\alpha=1$ one finds that the branch. cuts of $G(E)$ and $G^{(1)}(E)$ coincide on $-2 \leqq E \leqq 2$ and both $\mathscr{J}$ and $\mathscr{J}^{(1)}$ have only extended states. In this case

$$
\mathscr{J}=\left(\begin{array}{cccccc}
0 & 2 & & & & \\
2 & 0 & 1 & & & \\
& 1 & 0 & 1 & & \\
& & 1 & 0 & 1 \\
& & \ddots & \ddots & \ddots
\end{array}\right) \text { and } \mathscr{J}^{(1)}=\left(\begin{array}{lllll}
0 & 1 & & & \\
1 & 0 & 1 & & \\
& 1 & 0 & 1 & \\
& & \ddots & \ddots & \ddots
\end{array}\right)
$$

and gives rise to the Chebychev polynomials of the first and second kind respectively. For $\alpha>1$ we see from above that $\mathscr{J}^{(1)}$ has only localized states and

$$
G^{(1)}(E)=\sum_{j=0}^{\infty} \sum_{k=1}^{2^{j}} \frac{\Gamma_{k}^{(j)}}{z-z_{k}^{(j)}},
$$

since $Q(z)=z$. Equation (IV.1) implies that $G^{(1)}(E)$ does not have a branch cut. However, since du is the equilibrium measure associated with the Julia set (a Cantor set in this case), it is known that $\mathscr{J}$ has only extended states.

It has also been pointed out to us (Bellissard, private comm.) that the eigenstates of $\mathscr{J}^{(1)}$ may be considered as surface states [30].

Acknowledgements. We would like to thank Daniel Bessis and Pierre Moussa for valuable discussions pertaining to Sect. II. We would also like to thank J. Bellissard for pointing out the physical consequences of this work.

\section{References}

1. Julia, G.: Memoire sur l'iteration des fonctions rationelles. J. Math. Pures Appl. Ser. 8.1, 47-245 (1918)

2. Fatou, P.: Sur les equations fonctionelles. Bull. Soc. Math. France 47, 161-271 (1919), 48, 33-94, 208-314 (1920) 
3. Brolin, H.: Invariant sets under iteration of rational functions. Ark. Mat. 6, 103-144 (1965)

4. Barnsley, M. F., Geronimo, J. S., Harrington, A. N.: Orthogonal polynomial associated with invariant measures on Julia sets. Bull. A. M. S. 7, 381-384 (1982)

5. Bessis, D., Moussa, P.: Orthogonality properties of iterated polynomial mappings. Commun. Math. Phys. 88, 503-529 (1983)

6. Pitcher, T.S., Kinney, J. R.: Some connections between ergodic theory and the iteration of polynomials. Ark. Mat. 8, 25-32 (1969)

7. Barnsley, M. F., Harrington, A. N.: Moments of balanced measures on Julia sets. Trans. A. M. S. 284, 271-280 (1984)

8. Barnsley, M. F., Geronimo, J. S., Harrington, A. N.: Infinite dimensional Jacobi matrices associated with Julia sets. Proc. A.M.S. 88, 625-630 (1983)

9. Bellissard, J., Bessis, D., Moussa, P.: Chaotic states of almost periodic Schrödinger operators. Phys. Rev. Lett. 49, 701-704 (1982)

10. Bellissard, J.: Almost random operators, $k$-theory and spectral properties, CNRS Luminy (preprint)

11. Avron, J., Simon, B.: Singular continuous spectrum for a class of almost periodic Jacobi matrices. Bull. A.M.S. 6, 81-85 (1982)

12. Sarnak, P.: Spectral behavior of quasi-periodic potentials. Commun. Math. Phys. 84, 377-401 (1982)

13. Pasture, L. A.: Spectrum properties of disordered systems in the one-body approximation. Commun. Math. Phys. 75, 179-196 (1980)

14. Craig, W.: Pure point spectrum for discrete almost periodic Schrödinger operators. Commun. Math. Phys. 88, 113-131 (1981)

15. Simon, B.: Kotani theory for one dimensional stochastic Jacobi matrices. Commun. Math. Phys. 89, 227-234 (1983)

16. Delyon, F., Souillard, B.: The rotation number for finite difference operators and its properties. Commun. Math. Phys. 89, 415 (1983)

17. Barnsley, M. F., Geronimo, J. S., Harrington, A. N.: Geometry, electrostatic measure and orthogonal polynomials on Julia sets for polynomials. J. Erg. Theo. Dyn. Sys. (to appear)

18. Bessis, D., Geronimo, J. S., Moussa, P.: in preparation

19. Bessis, D., Geronimo, J. S., Moussa, P.: Mellin transforms associated with Julia sets and physical applications. J. Stat. Phys. (to appear)

20. Szego, G.: Orthogonal polynomials. Am. Math. Soc. Colloq. Publ. Vol. 23, Am. Math. Soc., Providence, RI (1939)

21. Besicovitch, A. S.: Almost periodic functions. Cambridge: Cambridge University Press 1932

22. Bessis, D., Mehta, M. L., Moussa, P.: Orthogonal polynomials on a family of Cantor sets and the problem of iterations of quadratic mappings. Lett. Math. Phys. 6, 123-140 (1982)

23. Reed, M., Simon, B.: Methods of modern mathematical physics: Functional analysis, p. 182 New York: Academic Press, 1972

24. Sell, G. R.: Topological dynamics and ordinary differential equations. New York: van Nostrand Reinhold, 1971

25. Barnsley, M. F., Geronimo, J. S., Harrington, A. N.: Georgia Institute of Technology, reprint (1983)

26. Anderson, P. W.: Absence of diffusion in certain random lattices. Phys. Rev. 109, 1492 (1958)

27. Economou, E. H., Cohen, M. H.: Localization in one-dimensional disordered systems. Phys. Rev. B4, 396 (1972)

28. Economou, E. H., Cohen, M. H.: Existence of mobility edges in Anderson's model for random lattices. Phys. Rev. B5, 2931 (1972)

29. Sokoloff, J. B.: Electron localization in crystals with quasiperiodic lattice potentials. Phys. Rev. B22, 5823 (1980)

30. Rammel, R.: The spectrum of harmonic excitations on fractals. J. Phys. 45, 191 (1984)

Communicated by B. Simon

Received February 1, 1984, in revised form December 14, 1984 
